Check for updates

Cite this: RSC Adv., 2018, 8, 18243

\title{
Fiber optic magnetic field sensor using Co doped ZnO nanorods as cladding
}

\begin{abstract}
S. Narasimman, ${ }^{a}$ L. Balakrishnan (D) *b and Z. C. Alex*a
A fiber optic magnetic field sensor is proposed and experimentally demonstrated. Pristine and Co doped ZnO nanorods of different Co concentrations (5, 10, 15 and 20 at\%) were synthesized using a hydrothermal method. The synthesized nanorods were subjected to various characterization methods like X-ray diffraction (XRD), optical absorption, scanning electron microscopy, energy dispersive X-ray spectroscopy, Fourier transform infrared spectroscopy, vibrating sample magnetometry and $X$-ray photoelectron spectroscopy (XPS). XRD and XPS analysis confirms that the Co ions were successfully incorporated into the $\mathrm{Zn}$ site of the wurtzite $\mathrm{ZnO}$ lattice without altering the structure. The pristine and Co doped $\mathrm{ZnO}$ nanorods showed remarkable changes in the $\mathrm{M}-\mathrm{H}$ loop where the diamagnetic behavior of $\mathrm{ZnO}$ changes to paramagnetic when doped with Co. The sensor structure is composed of cladding modified fiber coated with Co doped $\mathrm{ZnO}$ nanorods as a sensing material. The modified cladding is proportionally sensitive to the ambient magnetic field because of the magneto-optic effect. Experimental results revealed that the sensor has an operating magnetic field range from $17 \mathrm{mT}$ to 180 $\mathrm{mT}$ and shows a maximum sensitivity of $\sim 18 \%$ for 15 at\% Co doped ZnO nanorods. The proposed magnetic field sensor would be attractive due to its low cost fabrication, simplicity of the sensor head preparation, high sensitivity and reproducibility.
\end{abstract}

Received 1st March 2018

Accepted 24th April 2018

DOI: $10.1039 / \mathrm{c} 8 \mathrm{ra01803k}$

rsc.li/rsc-advances doped $\mathrm{ZnO}$ nanostructures and assessed their performance on doping. For instance, Sharma et al. have synthesized Co: ZnO nanoparticles using a co-precipitation method and showed their ferromagnetism at room temperature. ${ }^{10} \mathrm{Xu}$ et al. have synthesized Co doped ZnO nanoflakes using a hydrothermal method and noticed their ferromagnetism at room temperature. However, with increasing $\mathrm{Co}^{2+}$ dopant concentration, paramagnetism was exhibited. ${ }^{11}$ Qiu et al. have also reported room temperature ferromagnetism (RTFM) in Co: ZnO by water-bubble template process. ${ }^{12}$

In the past few years, several techniques have been ratified to synthesize pristine $\mathrm{ZnO}$ and $\mathrm{TM}$ doped $\mathrm{ZnO}$ nanostructures including co-precipitation, ${ }^{13}$ hydrothermal, ${ }^{14}$ sol-gel, ${ }^{15}$ magnetron sputtering, ${ }^{16}$ ball milling, ${ }^{17}$ etc. Of these, the hydrothermal method offers various nanostructure morphologies, controlled particle size, high reaction rate, different phase formation, etc. ${ }^{18}$ To explore the potential of nanoparticles in the field of magnetic field sensing, various types of sensor such as thin films, ${ }^{19}$ fiber optics ${ }^{20}$ Hall effect based magnetic field sensors and magnetic switches have been fabricated. Though, fiber optic based magnetic sensors offer numerous advantages such as small size, on-line analysis, remote sensing, high sensitivity, immunity to electromagnetic interference and a capability of working in harsh environments. ${ }^{21}$ In fiber optic sensor, the principle of sensing is based on cladding modification technology, in which the middle part of the cladding was replaced with a magnetic sensing material. The magnetic field sensing 
material causes the magneto-optic effect such as change in refractive index as a result of an applied external magnetic field.

Hitherto, various kinds of fiber optic based magnetic sensor have been proposed using magnetic fluid (MF) as the cladding with different optical devices such as Fiber Bragg Grating (FBG), ${ }^{22}$ Long Period Fiber Bragg Grating (LPFG), ${ }^{23}$ singlemode-multi-mode-single-mode (SMS) structures, ${ }^{24}$ multi-

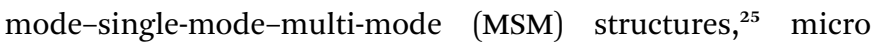
resonators, ${ }^{26}$ and Fabry-Pérot devices. ${ }^{27}$ However, minor shortcomings like the feeble response of MF to external magnetic fields, low interaction between core mode/lower order cladding mode and MF, structure instability and complicated technology mean that they fail to achieve higher magnetic sensitivity.

To overcome these shortcomings, a fiber optic magnetic field sensor based on Co doped $\mathrm{ZnO}$ nanorods is proposed and demonstrated. The impact of doping concentration on structural, optical and magnetic properties is also investigated.

\section{Materials and methods}

\subsection{Synthesis of pristine and Co doped $\mathrm{ZnO}$ nanorods}

Pristine $\mathrm{ZnO}$ and $\mathrm{Zn}_{1-x} \mathrm{Co}_{x} \mathrm{O}(x=5,10,15$ and 20 at\%) were synthesized using a hydrothermal method. All the chemicals used in this work were analytical reagent grade and used as received without further purification. Zinc acetate dihydrate $\left(\mathrm{Zn}\left(\mathrm{CH}_{3} \mathrm{COO}\right)_{2} \cdot 2 \mathrm{H}_{2} \mathrm{O}\right.$, 99.99\%, Sigma-Aldrich) and cobalt(II) chloride, (99.99\%, Sigma-Aldrich) in suitable weight percentages were used as starting materials. Additionally, sodium hydroxide ( $\mathrm{NaOH}, 99.99 \%$, Alfa Aesar) was used as the precipitating agent and cetyl trimethyl ammonium bromide (CTAB) $\left(\mathrm{C}_{19} \mathrm{H}_{42} \mathrm{BrN}\right)$ was used as a surfactant.

For the synthesis of pristine $\mathrm{ZnO}$ nanorods, $50 \mathrm{ml}$ of $0.2 \mathrm{M}$ zinc acetate dihydrate, $50 \mathrm{ml}$ of $0.1 \mathrm{M} \mathrm{CTAB}$ and $100 \mathrm{ml}$ of $2 \mathrm{M}$ $\mathrm{NaOH}$ aqueous solution were prepared. Initially, zinc acetate dihydrate solution was stirred continuously for $3 \mathrm{~h}$ and aqueous $\mathrm{NaOH}$ solution was added dropwise to the mixture until the $\mathrm{pH}$ value of solution reaches 8.0. Later, the white precipitated solution was stirred for $1 \mathrm{~h}$. Finally, CTAB solution was added into the mixture and stirred vigorously for another $30 \mathrm{~min}$. Then, the final solution was transferred into a $100 \mathrm{ml}$ teflonlined autoclave and maintained at $170{ }^{\circ} \mathrm{C}$ for $72 \mathrm{~h}$. After the reaction was completed, the resultant product was washed several times with distilled water and ethanol alternatively, and dried at $60{ }^{\circ} \mathrm{C}$ overnight.

For the synthesis of $\mathrm{Zn}_{1-x} \mathrm{Co}_{x} \mathrm{O}(x=5,10,15$ and 20 at\%) nanorods, the calculated amount of cobalt(II) chloride and zinc acetate dihydrate were dissolved in $50 \mathrm{ml}$ of distilled water and stirred for $3 \mathrm{~h}$. Then, aqueous $\mathrm{NaOH}$ solution was added drop wise into the above mixture to form a white precipitate with a slight pale pink colour. After that the same procedure that was adopted for the synthesis of pristine $\mathrm{ZnO}$ nanorods was followed for the synthesis of Co doped $\mathrm{ZnO}$ nanorods.

\subsection{Sensor head preparation and setup}

Fig. 1(a) shows the schematic diagram of the proposed magnetic field sensor structure. A multi-mode plastic optical
$\mathrm{Zn}_{1-\mathrm{x}} \mathrm{Co}_{\mathrm{x}} \mathrm{O} \mathrm{NR}(\mathrm{x}=0.05,0.1,0.15$ and 0.2$)$

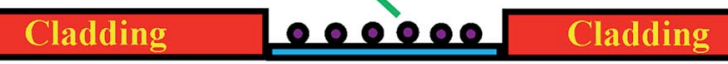

Core

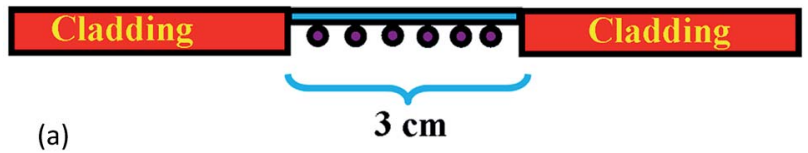

(b)

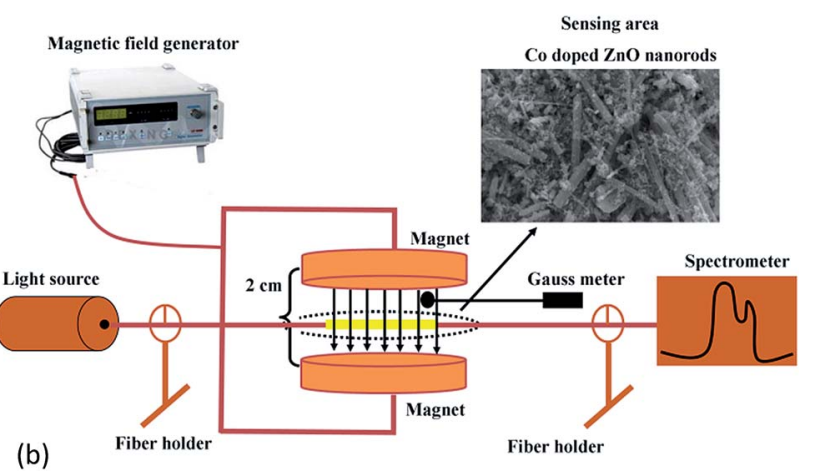

Fig. 1 (a) Schematic diagram of the proposed magnetic field sensing structure. (b) Experimental setup for the magnetic field sensor.

fiber of about $42 \mathrm{~cm}$ length with $1000 \mu \mathrm{m}$ diameter and 0.51 numerical aperture was used as a sensor head. A cladding region of about $3 \mathrm{~cm}$ in length was mechanically removed at the center portion of the fiber using a chemical etching process followed by fine polishing. The ablated cladding region was chemically etched using acetone followed by polishing with a 1000 grid sheet. Then, the polished surface was cleaned and coated with Co (at\% of 5, 10, 15 and 20) doped ZnO nanorods using a dip coating method.

The scheme of the experimental setup used for measuring the magnetic field sensing characteristics of the fabricated sensor is shown in Fig. 1(b). In the sensor setup, a broadband light source (halogen lamp-SLS201/M) with the wavelength ranging from 300 to $2600 \mathrm{~nm}$ is coupled at one end of the fiber and the intensity spectrum was recorded with a fiber optic spectrometer (CCS200/M) having a spectral range of 200 to $1000 \mathrm{~nm}$ at the other end of the fiber. The sensor head was inserted between the two poles of an electromagnet which generates a static magnetic field around the sensing head. The experiment was conducted at ambient temperature $\left(28^{\circ} \mathrm{C}\right)$.

\section{Results and discussion}

\subsection{Structural analysis}

5, 10, 15 and 20 at\% Co doped $\mathrm{ZnO}$ nanorods are represented as ZC1, ZC2, ZC3 and ZC4 respectively.

$\mathrm{XRD}$ patterns of pristine $\mathrm{ZnO}$ and $\mathrm{Zn}_{1-x} \mathrm{Co}_{x} \mathrm{O}(x=0.05,0.1$, 0.15 and 0.2 ) nanorods are shown in Fig. 2. All the diffraction peaks from the XRD patterns clearly manifest that the $\mathrm{Zn}_{1-x^{-}}$ $\mathrm{Co}_{x} \mathrm{O}$ nanorods addresses the hexagonal wurtzite structure and 


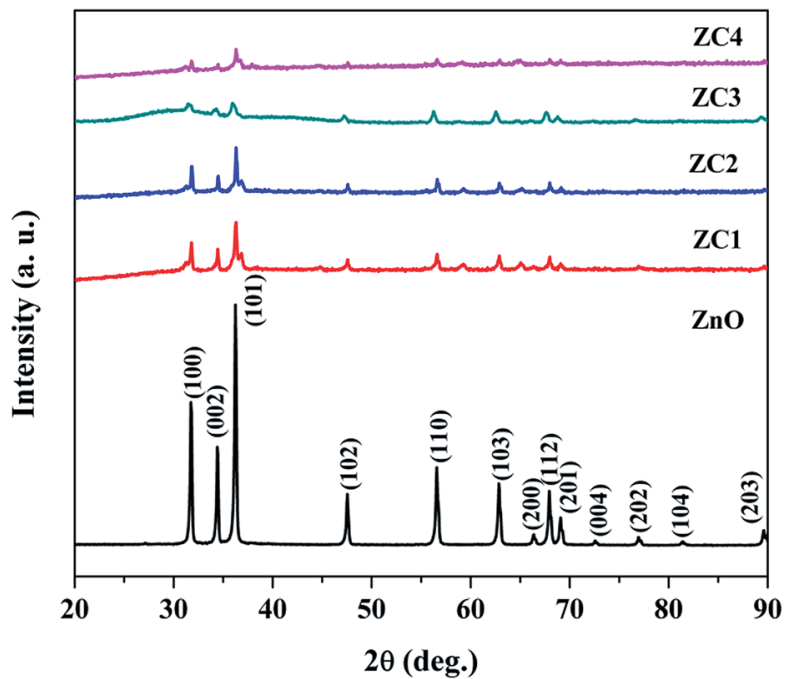

Fig. 2 XRD pattern of pristine $\mathrm{ZnO}$ and $\mathrm{Zn}_{1-x} \mathrm{Co}_{x} \mathrm{O}$ nanorods.

are very well matched with standard JCPDS card no: 36-1451. No other secondary phases of Co clusters were found. The nonexistence of impurity peaks reveal that Co ions were successfully incorporated into the $\mathrm{ZnO}$ lattice. The decrease in diffraction peak intensity is due to an increase in the concentration of impurities. ${ }^{28}$ The average crystallite size and strain of the nanorods are calculated from the Williamson and Hall (WH) plot. $^{29}$

The average crystallite size and strain of the nanoparticles are shown in Table 1. It was found that due to the incorporation of impurities, the crystallite size decreases.

\subsection{Morphological and elemental analysis}

Fig. 3(a-j) shows the morphologies of pristine and $\mathrm{Zn}_{1-x} \mathrm{Co}_{x} \mathrm{O}$ nanoparticles at different magnifications. It is seen that the synthesized nanopowder shows a rod like morphology. In order to analyse the complete morphology information of the $\mathrm{ZnO}$ nanorods, a plausible formation mechanism for the $\mathrm{ZnO}$ nanorods was proposed, as schematically illustrated in Fig. 4.

The formation mechanism of Co doped $\mathrm{ZnO}$ nanorods can be invoked via the following chemical reactions:

Decomposition of $\mathrm{Zn}(\mathrm{Ac})_{2}$ :

$$
\begin{aligned}
\mathrm{Zn}\left(\mathrm{CH}_{3} \mathrm{COO}\right)_{2} \cdot 2 \mathrm{H}_{2} \mathrm{O}+2 \mathrm{NaOH} \rightarrow \\
\mathrm{Zn}(\mathrm{OH})_{2}+2 \mathrm{CH}_{3} \mathrm{COONa}+2 \mathrm{H}_{2} \mathrm{O}
\end{aligned}
$$

Table 1 Crystallite size and microstrain of pristine $\mathrm{ZnO}$ and $\mathrm{Zn}_{1-x} \mathrm{Co}_{x} \mathrm{O}$ nanorods

\begin{tabular}{lll}
\hline Sample & $\begin{array}{l}\text { Crystallite size } \\
(D)(\mathrm{nm})\end{array}$ & $\begin{array}{l}\text { Microstrain } \\
(\varepsilon)\end{array}$ \\
\hline ZnO & 65 & 0.00070 \\
ZC1 & 63 & 0.00073 \\
ZC2 & 57 & 0.00076 \\
ZC3 & 53 & 0.00082 \\
ZC4 & 49 & 0.00085
\end{tabular}
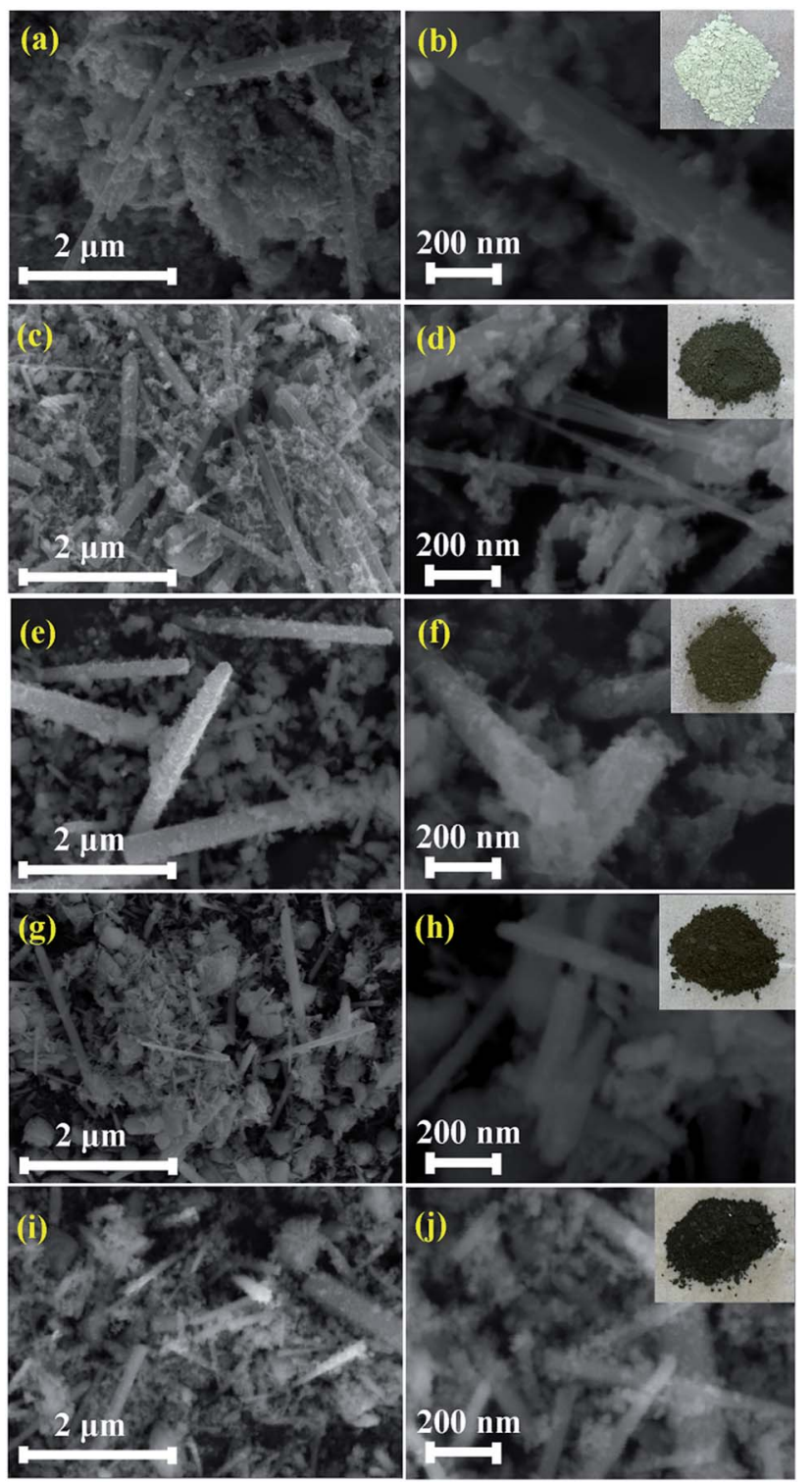

Fig. 3 SEM images of pristine $\mathrm{ZnO}$ and $\mathrm{Zn}_{1-x} \mathrm{Co}_{x} \mathrm{O}$ nanorods.

Production of pristine $\mathrm{ZnO}$ nanoparticles:

$$
\begin{gathered}
\mathrm{Zn}(\mathrm{OH})_{2} \leftrightarrow \mathrm{Zn}^{2+}+2 \mathrm{OH}^{-} \\
\mathrm{Zn}(\mathrm{OH})_{2}+2 \mathrm{OH}^{-} \rightarrow\left[\mathrm{Zn}(\mathrm{OH})_{4}{ }^{2-}\right] \\
{\left[\mathrm{Zn}(\mathrm{OH})_{4}{ }^{2-}\right] \rightarrow \mathrm{ZnO}+\mathrm{OH}^{-}+\mathrm{H}_{2} \mathrm{O}}
\end{gathered}
$$

Decomposition of $\mathrm{CoCl}_{2}$ :

$$
\begin{gathered}
\mathrm{CoCl}_{2}+2 \mathrm{NaOH} \rightarrow \mathrm{Co}(\mathrm{OH})_{2}+2 \mathrm{NaCl} \\
\mathrm{Co}(\mathrm{OH})_{2} \leftrightarrow \mathrm{Co}^{2+}+2 \mathrm{OH}^{-}
\end{gathered}
$$

Production of Co doped ZnO nanoparticles:

$$
\mathrm{Zn}_{1-x}(\mathrm{OH})_{2}+\mathrm{Co}_{x}(\mathrm{OH})_{2} \rightarrow \mathrm{Zn}_{1-x} \mathrm{Co}_{x} \mathrm{O}+\mathrm{H}_{2} \mathrm{O}
$$




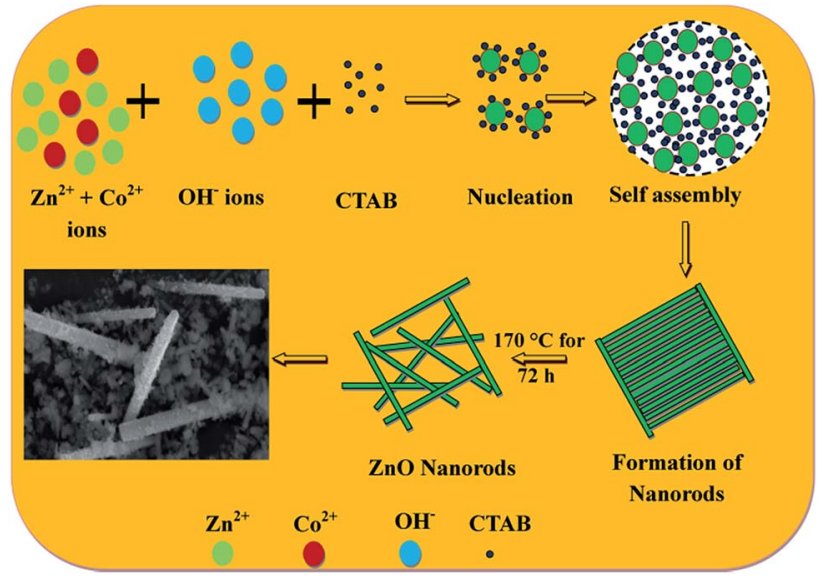

Fig. 4 Schematic formation mechanism of $\mathrm{Zn}_{1-x} \mathrm{Co}_{x} \mathrm{O}$ nanorods.

Reaction (1) and (5) shows the decomposition of $\mathrm{Zn}(\mathrm{Ac})_{2}$ and $\mathrm{CoCl}_{2}$ in an ambient environment producing $\mathrm{Zn}(\mathrm{OH})_{2}$ and $\mathrm{Co}(\mathrm{OH})_{2}$, respectively. If the $\mathrm{pH}$ value in aqueous solution is about 11 , where $\mathrm{Zn}(\mathrm{OH})_{2}$ is the main chemical compound, during the hydrothermal method, a part of the $\mathrm{Zn}(\mathrm{OH})_{2}$ colloid species dissolves into $\mathrm{Zn}^{2+}$ and $\mathrm{OH}^{-}$according to reaction (2). When the concentration of $\mathrm{Zn}^{2+}$ and $\mathrm{OH}^{-}$reaches the super saturation degree of $\mathrm{ZnO}$, then $\mathrm{ZnO}$ nuclei will form according to reaction (3) and (4). Reaction (6) indicates the dissolution of $\mathrm{Co}(\mathrm{OH})_{2}$ in water to produce $\mathrm{Co}^{2+}$ ions that will be incorporated into the $\mathrm{ZnO}$ lattice. Reaction (7) is the final step of the growth process to accomplish Co doped ZnO nanoparticles. ${ }^{30}$ To obtain the consistent nanoparticle size, the headway approach to synthesize Co doped $\mathrm{ZnO}$ nanoparticles using a hydrothermal method is closely related to a new understanding of the formation mechanism by the introduction of the surfactant CTAB. CTAB is a cationic surfactant having small hydrophilic head and a hydrophobic tail. The element $\mathrm{Zn}$ was obtained in $\left[\mathrm{Zn}(\mathrm{OH})_{4}\right]^{2-}$ as a negatively charged tetrahedrons that were formed according to reaction (3), whereas $\mathrm{CTA}^{+}$was positively charged with a tetrahedral head. At a higher reaction temperature $\left(\sim 170{ }^{\circ} \mathrm{C}\right)$, the cationic charge of $\mathrm{CTA}^{+}$and anionic charge of $\left[\mathrm{Zn}(\mathrm{OH})_{4}\right]^{2-}$ were formed primarily by electrostatic interactions (reaction (4)). The CTAB could accelerate the ionization of $\left[\mathrm{Zn}(\mathrm{OH})_{4}\right]^{2-}$ as it is a strong acid-weak-base salt. ${ }^{31} \mathrm{It}$ was assumed that the CTAB was aggregated in between the $\mathrm{ZnO}$ crystallites during hydrothermal crystallization and after washing thrice with ethanol, the rod like structure of $\mathrm{ZnO}$ was formed.

The elemental composition of pristine and $\mathrm{Zn}_{1-x} \mathrm{Co}_{x} \mathrm{O}$ nanorods were investigated using EDS spectra and shown in Fig. 5(a-e). The spectra affirm the presence of $\mathrm{Zn}$ and $\mathrm{O}$ in $\mathrm{ZnO}$, likewise, $\mathrm{Zn}$, $\mathrm{O}$ and $\mathrm{Co}$ in Co doped ZnO nanorods. Fig. 6 shows the elemental mapping of $\mathrm{Zn}$, O and Co on a single Co doped $\mathrm{ZnO}$ nanorod. The mapping results indicated that $\mathrm{Zn}, \mathrm{O}$ and $\mathrm{Co}$ atoms are uniformly distributed on the single nanorod.

\subsection{FTIR analysis}

Fig. 7 shows the vibrational frequencies of pristine and Co doped ZnO nanorods. The peak obtained between 3420$3650 \mathrm{~cm}^{-1}$ corresponds to the stretching vibration of $\mathrm{O}-\mathrm{H} .{ }^{32}$ The

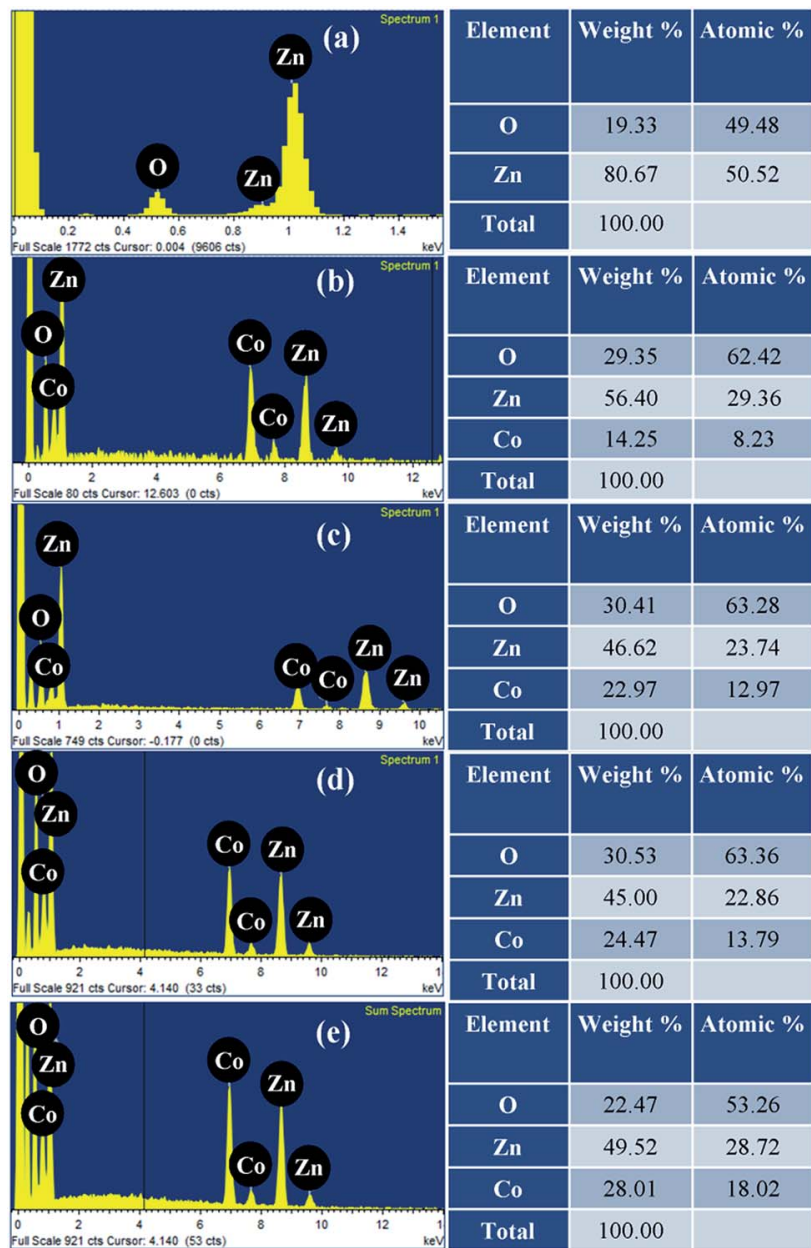

Fig. 5 EDS spectra of pristine $\mathrm{ZnO}$ and $\mathrm{Zn}_{1-x} \mathrm{Co}_{x} \mathrm{O}$ nanorods.

stretching vibrations of $\mathrm{Zn}-\mathrm{O}$ are observed at 414, 422, 428, 457 and $466 \mathrm{~cm}^{-1}$ for pristine and Co doped $\mathrm{ZnO}$ nanorods. ${ }^{33}$

Upon Co doping, the IR peak shifts consistently from $414 \mathrm{~cm}^{-1}$ to $466 \mathrm{~cm}^{-1}$ and this shift is attributed to the incorporation of $\mathrm{Co}^{2+}$ ions in the $\mathrm{ZnO}$ lattice. ${ }^{34}$
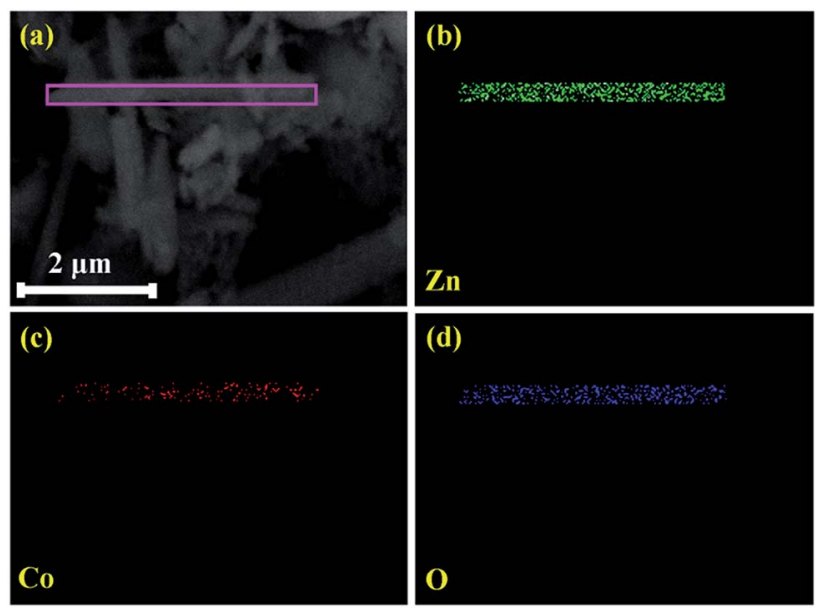

Fig. 6 Elemental mapping of ZC3 nanorods. 


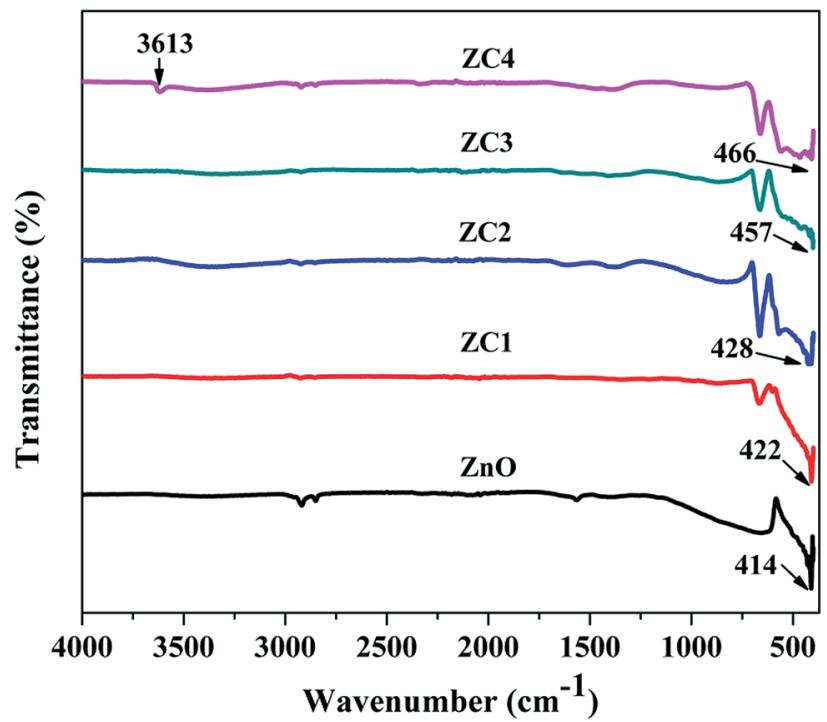

Fig. 7 FTIR spectrum of pristine and Co doped $\mathrm{ZnO}$ nanorods.

\subsection{Optical absorption studies}

Fig. 8 shows the optical absorption spectra of pristine and Co doped $\mathrm{ZnO}$ nanorods recorded in DRS mode. The absorption edge of the Co doped ZnO depicts significant red shift compared with pristine $\mathrm{ZnO}$. It is attributed that the Co doped $\mathrm{ZnO}$ nanorods showed absorption from UV to visible region due to the $\mathrm{d}-\mathrm{d}$ transitions. ${ }^{35}$ This affirms the incorporation of $\mathrm{Co}^{2+}$ ions into the $\mathrm{ZnO}$ lattice, rather than forming as cobalt oxide $(\mathrm{CoO})$ or Co metal.

\subsection{VSM analysis}

Fig. 9 shows magnetization versus magnetic field $(M-H)$ measurements with maximum applied field $\pm 10 \mathrm{kOe}$ at room temperature $(300 \mathrm{~K})$. All the Co doped $\mathrm{ZnO}$ nanorods revealed paramagnetic behaviour excluding pristine $\mathrm{ZnO}$ (diamagnetic behaviour). The diamagnetic behaviour of pristine $\mathrm{ZnO}$ is

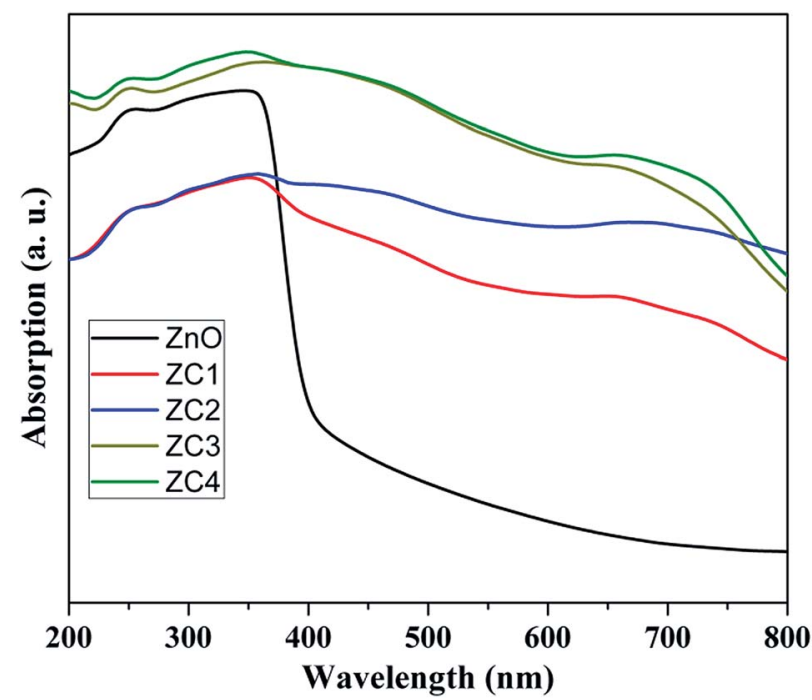

Fig. 8 Absorption spectrum of pristine and Co doped $\mathrm{ZnO}$ nanorods. ascribed to the presence of paired electrons in its d orbital. The $5 \%$ and $10 \%$ Co doped $\mathrm{ZnO}$ nanorods (ZC1 and ZC2) deduces weak ferromagnetism (Fig. 9(b)). It is suggested that a few of the

(a)

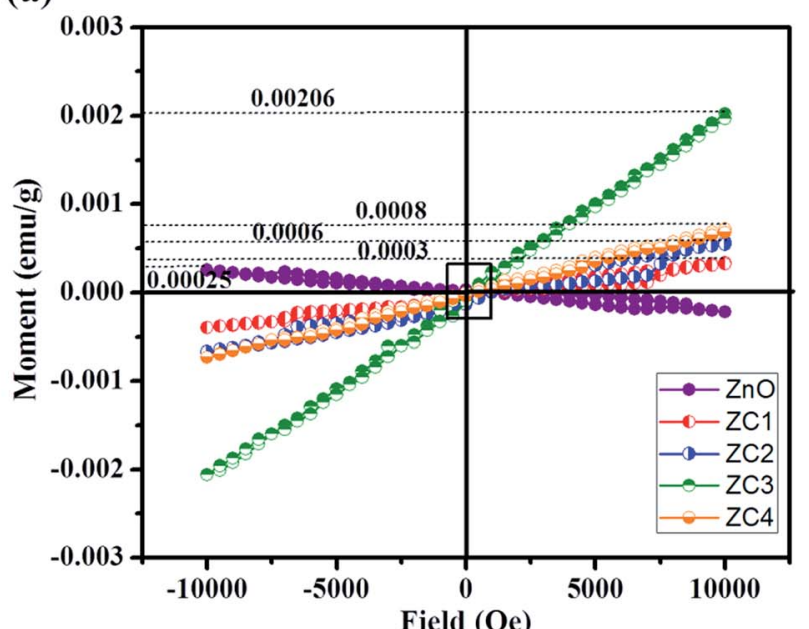

(b)

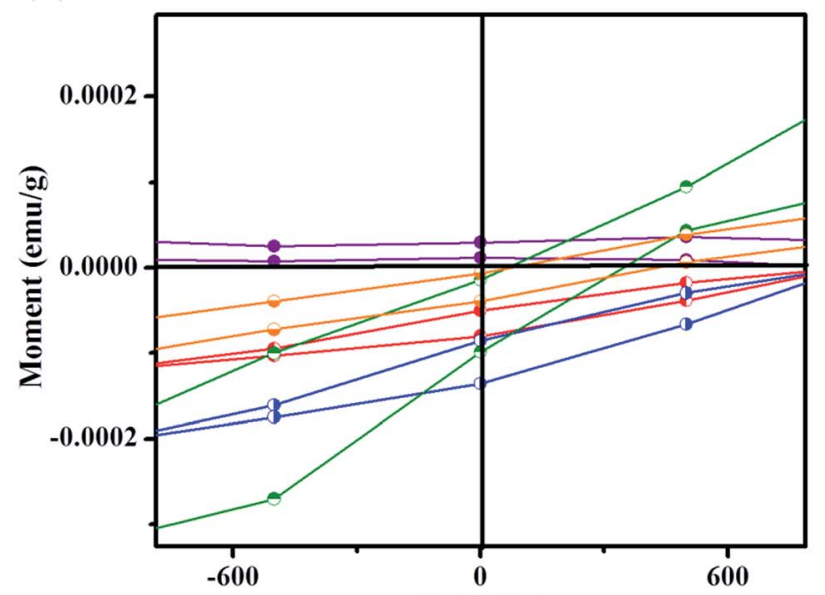

(c)

Field (Oe)

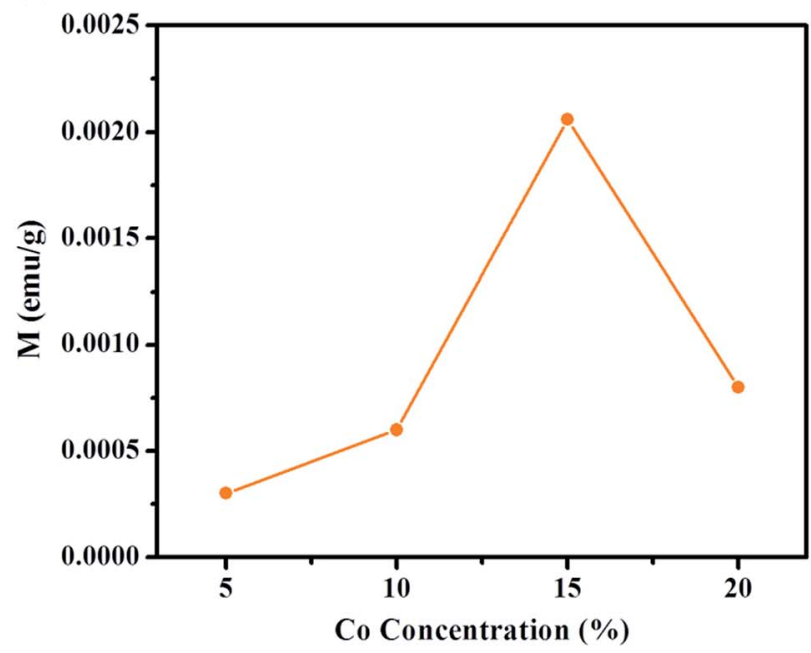

Fig. 9 (a) Magnetic field dependent magnetization curve of pristine $\mathrm{ZnO}$ and $\mathrm{Zn}_{1-x} \mathrm{Co}_{x} \mathrm{O}$ nanorods at $300 \mathrm{~K}$. (b) Zoomed magnetization plot and (c) variation of magnetization $M$ with Co concentration. 
doped $\mathrm{Co}^{2+}$ cations occupy the next nearest lattice sites. The nearest Co-Co pairs couple in an antiferromagnetic way and suppress the magnetization. ${ }^{36-39}$ Thus, weak ferromagnetism was observed in the ZC1 and ZC2 samples. When the level of Co doping increases to $20 \%$, the sample demonstrates linear magnetization curves with the absence of a hysteresis loop within the applied field (ZC3 and ZC4), which can be concluded as good paramagnetism. As Co dopant concentration increases, more and more nearest Co-Co pairs exhibit larger antiferromagnetic interactions, which leads to the vanishing of hysteresis in the ZC3 and ZC4 samples. This implies that higher Co doping concentrations in $\mathrm{ZnO}$ lead to paramagnetism. ${ }^{\mathbf{0}}$ Similar paramagnetic behaviour with the same composition has been reported by many research groups. ${ }^{41-45}$ However, for higher magnetizing fields the magnetization is found to increase with increasing Co concentration (Fig. 9(c)).

\subsection{XPS analysis}

Furthermore, to confirm the presence of elements and its chemical bonding states in synthesized Co doped $\mathrm{ZnO}$ nanorods $(x=0.15)$, XPS analysis has been carried out. Fig. 10(a) depicts the full range survey spectrum of Co doped $\mathrm{ZnO}$ nanorods $(x=0.15)$, which reveals the presence of characteristic peaks of $\mathrm{Zn}, \mathrm{O}$ and $\mathrm{Co}$ in the synthesized Co doped $\mathrm{ZnO}$ nanorods. The selected data was corrected with $\mathrm{C}$ 1s carbon contamination peak (284.6 eV). Fig. 10(b) shows the high resolution spectra of the $\mathrm{Zn} 2 \mathrm{p}$ energy state. The core level binding energy of $\mathrm{Zn} 2 \mathrm{p}_{3 / 2}$ and $\mathrm{Zn} 2 \mathrm{p}_{1 / 2}$ was observed at $1021.9 \mathrm{eV}$ and $1044.9 \mathrm{eV}$, respectively. The energy difference between these two peaks $(\sim 23 \mathrm{eV})$ confirms that $\mathrm{Zn}$ exists primarily in the $\mathrm{Zn}^{2+}$ chemical state. ${ }^{46}$ Fig. 10 (c) depicts three distinct characteristic peaks of the $\mathrm{O} 1 \mathrm{~s}$ energy state observed at $528.3 \mathrm{eV}, 530.1 \mathrm{eV}$ and $531.8 \mathrm{eV}$, which are ascribed to the formation of three different $\mathrm{O}$ species in the synthesized nanorods. The lower binding energy implies that lattice oxygen in hexagonal wurtzite structure is surrounded by zinc and cobalt ions. The medium binding energy affirms the presence of oxygen vacancies in the ZnO matrix. The higher binding energy attributes the formation of adsorbed oxygen $\left(\mathrm{O}^{2-}\right)$ on the surface of the $\mathrm{ZnO}$ nanorods. ${ }^{47}$

Fig. 10(d) shows the Co $2 \mathrm{p}$ energy state high resolution spectra. The two characteristic peaks of Co $2 \mathrm{p}_{3 / 2}$ and Co $2 \mathrm{p}_{1 / 2}$, located at $780.4 \mathrm{eV}$ and $796.4 \mathrm{eV}$, respectively, have been observed. In the high resolution spectra of Co $2 \mathrm{p}$, the energy difference between Co $2 \mathrm{p}_{3 / 2}$ and Co $2 \mathrm{p}_{1 / 2}$ energy states is $\sim 16 \mathrm{eV}$, clearly showing that Co was successfully incorporated as a divalent ion. This is very well matched with previous literature reports. ${ }^{4-50}$ In addition to this, two shake-up satellite peaks ( $\mathrm{S} 1$ and S2) were observed at $785 \mathrm{eV}$ and $796.4 \mathrm{eV}$ along with the main characteristic peaks of the Co $2 p$ energy state. Thus, XPS analysis clearly reveals that $\mathrm{Co}^{2+}$ was successfully incorporated into the $\mathrm{ZnO}$ lattice by substituting $\mathrm{Zn}^{2+}$ without any additional impurities or phases.

\subsection{Magnetic field sensing analysis}

3.7.1. Sensing mechanism. Fig. 11 shows the magnetic field sensing mechanism in the proposed fiber optic sensor. In (a)

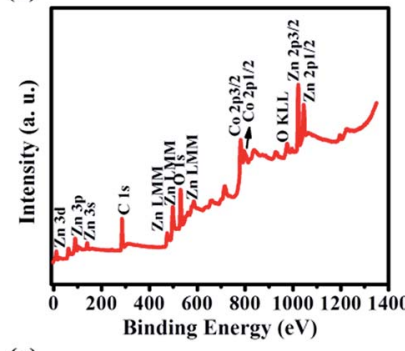

(c)

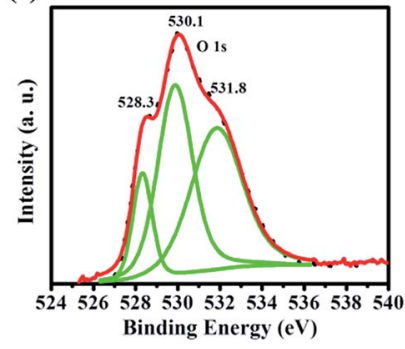

(b)

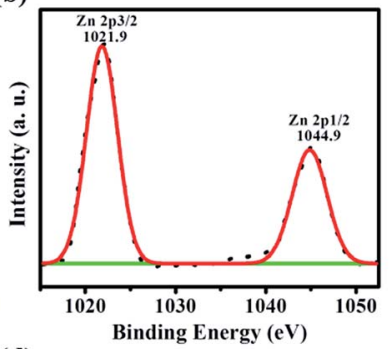

(d)

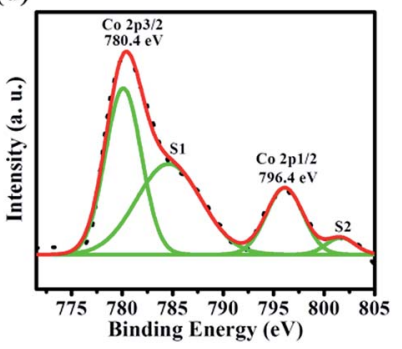

Fig. 10 (a) XPS survey spectrum of Co doped $\mathrm{ZnO}$ nanorods $(x=0.15)$. $(b-d)$ High resolution spectra of $\mathrm{Zn}, \mathrm{O}$ and Co, respectively.

the clad modified fiber optic sensor, the output light spectral variation with applied magnetic field leads the evanescent wave absorption in the modified cladding due to the change in the refractive index. When light travels with total internal reflection at the interface of the core and modified cladding, not all of the light intensity is reflected back but a part of it penetrates into the cladding material and its intensity decays exponentially away from the interface. This phenomenon is called an evanescent field. ${ }^{51}$ When an external magnetic field was subjected transversely to the direction of incident light and interacts with the evanescent field, the transmitted light spectrum and the output light intensity of the sensor varies. The reason for the change in intensity variation for the cladding modified fiber in an external magnetic field is based on the following relation, ${ }^{52-54}$

$$
\eta=\sqrt{\varepsilon_{\mathrm{r}}}=\sqrt{1+\chi}
$$

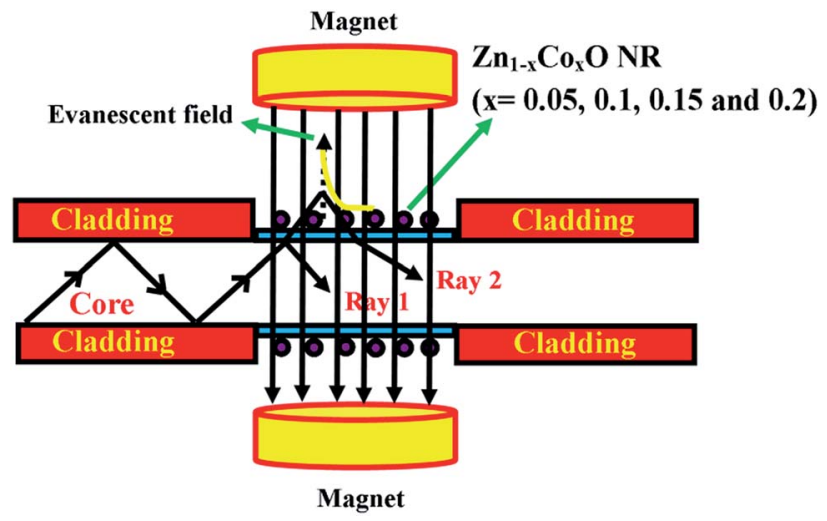

Fig. 11 Scheme of the magnetic field sensing mechanism in the proposed magnetic field sensor. 
From the equation, $\varepsilon_{\mathrm{r}}$ is the dielectric constant and $\chi$ is the electric susceptibility. When an external magnetic field is applied perpendicularly to the direction of the propagated light, then

$$
\frac{\partial \chi}{\partial H}>0
$$

So the refractive index of Co doped $\mathrm{ZnO}$ nanorods will increase with increasing in magnetic field strength. In the present work, a part of the cladding $\left(n_{\text {clad }}=1.402\right)$ was replaced with the synthesized pristine and Co doped $\mathrm{ZnO}$ nanorods $\left(n_{\mathrm{ZnO}}=1.91\right)$ which contributes to a certain amount of decreased attenuation in the guided signal depending on the absorbance of the cladding.

Hence, the change in refractive index and the absorbance of the cladding should affect the total internal reflection and evanescent field respectively. This is reflected in the change in intensity of the signal which is guided along the fiber..$^{55}$ The proposed fiber optic sensor works in a leaky mode condition as the refractive index of the modified cladding $\left(n_{\mathrm{Zno}}=1.91\right)$ is higher than that of the core $\left(n_{\text {core }}=1.492\right)$.

3.7.2. Spectral analysis. Fig. 12(a-e) shows the magnetic field sensing characteristics of pristine and Co doped $\mathrm{ZnO}$ nanorods with the magnetic field ranging from $17.2 \mathrm{mT}$ to 190.6 mT. The spectra exhibit three peaks around 693, 772 and $946 \mathrm{~nm}$ which are characteristic of the optical fiber used. These spectra suggest that the spectra only undergo intensity variation for different magnetic field strengths. It is clearly seen from the figure that the spectral intensity increases monotonically with increasing applied magnetic field strength. This is due to the decrease in evanescent wave absorption with increasing magnetic field. The light intensity in the absence of a magnetic field is taken as a reference.

3.7.3. Sensitivity analysis. The sensitivity of the proposed magnetic field sensor is calculated using the following relation, ${ }^{56}$

$$
S(\%)=\frac{\left|I_{\mathrm{m}}-I_{\mathrm{a}}\right|}{I_{\mathrm{a}}} \times 100
$$

where $I_{\mathrm{a}}$ is the intensity in the absence of a magnetic field and $I_{\mathrm{m}}$ is the intensity in the presence of a magnetic field.

Fig. 13 shows the magnetic field sensitivity plot of pristine and Co doped $\mathrm{ZnO}$ nanorods in different magnetic field ranging from $17.2 \mathrm{mT}$ to $190.6 \mathrm{mT}$ at an ambient temperature of $28^{\circ} \mathrm{C}$. From Fig. 13, it can be seen that Co doped ZnO nanorods $(x=$ $0.05,0.1,0.15$ and 0.2$)$ show enhanced sensitivity compared to pristine ZnO. Particularly, Co doped ZnO nanorods $(x=0.15)$ exhibit a maximum sensitivity of $\sim 18 \%$ compared with that of other Co doped ZnO nanorods ( $x=0.05,0.1$ and 0.2$)$. On the basis of the data plotted in Fig. 13, we can summarize that the sensor response increases fairly up to a Co doping of $x=0.15$, then decreases for higher doping concentrations. It is seen that the materials with higher magnetization possess high sensitivity.

Further, beyond the magnetic field of $180.8 \mathrm{mT}$, the sensor showed a saturated response. Therefore, the operating range for the proposed magnetic sensor is in the range of $17.2 \mathrm{mT}$ to $180.8 \mathrm{mT}$.
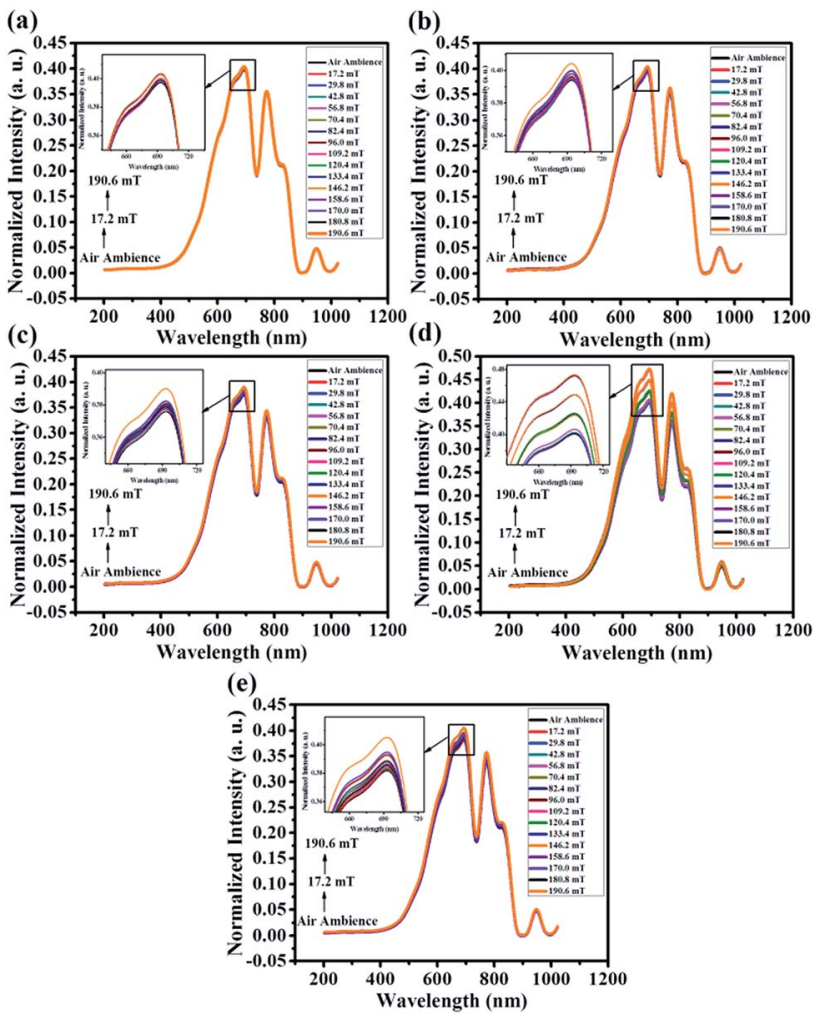

Fig. 12 Spectral response of the proposed sensor with different magnetic field strength ranging from $17.2 \mathrm{mT}$ to $190.6 \mathrm{mT}$ (a) $\mathrm{ZnO}$, (b) ZC1, (c) ZC2, (d) ZC3 and (e) ZC4.

In order to study the effect of reproducibility, the sensor was characterized over ten cycles with a magnetic field strength of $190 \mathrm{mT}$ and the experimental results are shown in Fig. 14. It is seen that the sensing response for all cycles are nearly identical with insubstantial sensitivity fluctuations. This clearly depicts that the sensor has good reproducibility.

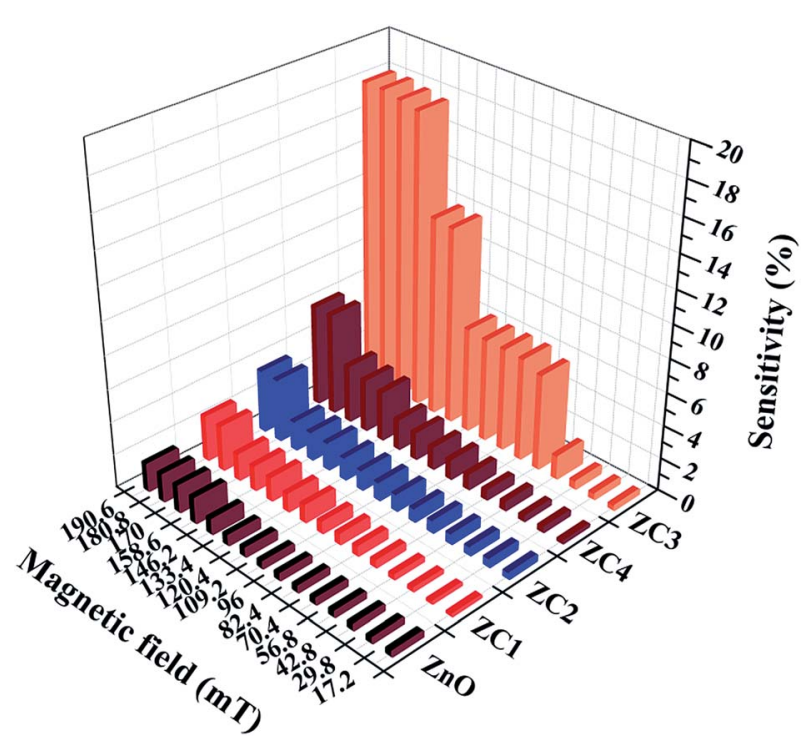

Fig. 13 Magnetic sensitivity of the proposed sensor with magnetic field strength ranging from $17.2 \mathrm{mT}$ to $190.6 \mathrm{mT}$. 


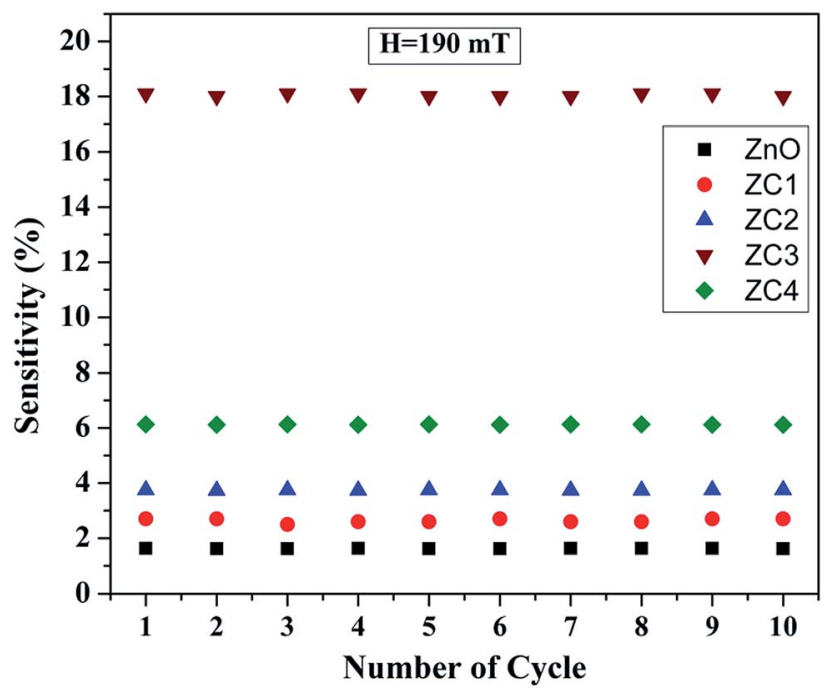

Fig. 14 Reproducibility plot of pristine $\mathrm{ZnO}$ and $\mathrm{Zn}_{1-x} \mathrm{Co}_{x} \mathrm{O}$ nanorods towards magnetic field strength of $190 \mathrm{mT}$.

\section{Conclusions}

A fiber optic magnetic field sensor using Co doped ZnO nanorods $(5,10,15$ and 20 at\%) has been proposed and experimentally demonstrated. The analysis confirms that the nanorods are in hexagonal wurtzite structure. Furthermore, the VSM analysis indicates that the Co doped ZnO nanorods exhibit weak ferromagnetism at lower doping levels (ZC1 and ZC2) and paramagnetism at higher doping levels (ZC3 and ZC4). Experimental results show that the sensor has an operating magnetic field range from $17.2 \mathrm{mT}$ to $180.8 \mathrm{mT}$ and revealed the maximum sensitivity of $\sim 18 \%$ for Co doped $\mathrm{ZnO}$ nanorods $(x=$ $0.15)$ which shows higher value of magnetization. The proposed magnetic field sensor will serve as a better platform for the development of fiber optic magnetic field sensors as better replacements for current Hall effect based sensors.

\section{Conflicts of interest}

There are no conflicts to declare.

\section{Acknowledgements}

The authors would like to acknowledge the Department of Science and Technology (DST), New Delhi, India for providing financial support through the FIST (Fund for Improvement of S\&T Infrastructure in Higher Educational Institutions) project [SR/FST/ETI-015/2011].

\section{References}

1 A. Kołodziejczak-Radzimska and T. Jesionowski, Materials, 2014, 7, 2833.

2 V. Avrutin, D. Silversmith and H. Morkoc, Proc. IEEE, 2010, 98, 1269.
3 U. Ozgür, Y. I. Alivov, C. Liu, A. Teke, M. A. Reshchikov, S. Dogan, V. Avrutin, S. J. Cho and H. Morkoc, J. Appl. Phys., 2005, 98, 041301.

4 D. S. Bohle and C. J. Spina, J. Phys. Chem. C, 2010, 114, 18139.

5 L. Yan, F. Zhao, S. Li, Z. Hu and Y. Zhao, Nanoscale, 2011, 3, 362.

6 S. Fabbiyola, L. John Kennedy, U. Aruldoss, M. Bououdina, A. A. Dakhel and J. J. Vijaya, Powder Technol., 2015, 286, 757.

7 G. Vijayaprasath, G. Ravi, A. S. Haja Hameed and T. Mahalingam, J. Phys. Chem. C, 2014, 118, 9715.

8 M. Shatnawi, A. M. Alsmadi, I. Bsoul, B. Salameh, G. A. Alna'washi, F. Al-Dweri and F. El Akkad, J. Alloys Compd., 2016, 655, 244.

9 M. Jay Chithra, K. Pushpanathan and M. Loganathan, Mater. Manuf. Processes, 2014, 29, 771.

10 P. K. Sharma, R. K. Dutta and A. C. Pandey, J. Colloid Interface Sci., 2010, 345, 149.

11 X. Y. Xu and C. B. Cao, J. Alloys Compd., 2010, 501, 265.

12 Y. C. Qiu, W. Chen, S. H. Yang, B. Zhang, X. X. Zhang, Y. C. Zhong and K. S. Wong, Cryst. Growth Des., 2010, 10, 177.

13 P. Geetha Devi and A. Sakthi Velu, J. Theor. Appl. Phys., 2016, 10, 233.

14 T. T. Loan, N. N. Long and L. H. Ha, J. Phys. D: Appl. Phys., 2009, 42, 065412.

15 J. El Ghoul, M. Kraini, O. M. Lemine and L. El Mir, J. Mater. Sci.: Mater. Electron., 2015, 26, 2614.

16 Y. Jouane, S. Colis, G. Schmerber, P. Kern, A. Dinia, T. Heiser and Y. A. Chapuis, J. Mater. Chem., 2011, 21, 1953.

17 B. Pal and P. K. Giri, J. Nanosci. Nanotechnol., 2011, 11, 1.

18 K. Shingange, Z. P. Tshabalala, O. M. Ntwaeaborwa, D. E. Motaung and G. H. Mhlongo, J. Colloid Interface Sci., 2016, 479, 127.

19 F. Pan, C. Song, X. J. Liu, Y. C. Yang and F. Zeng, Mater. Sci. Eng., $R, 2008,62,1$.

20 X. Lei, J. Chen, F. Shi, D. Chen, Z. Ren and B. Peng, Opt. Commun., 2016, 374, 76.

21 L. Luo, S. Pu, S. Dong and J. Tang, Sens. Actuators, A, 2015, 236, 67.

22 C. Li, T. Ning, X. Wen, J. Li, C. Zhang and C. Zhang, Opt. Laser Technol., 2015, 72, 104.

23 Z. Gu, Q. Ling, J. Lan and K. Gao, Opt. Laser Technol., 2017, 96, 249.

24 L. Luo, S. Pu, S. Dong and J. Tang, Sens. Actuators, A, 2015, 236, 67.

25 B. Yin, Y. Li, Z. Liu, S. Feng, Y. Bai, Y. Xu and S. Jian, Opt. Laser Technol., 2016, 80, 16.

26 X. Li and H. Ding, Opt. Lett., 2012, 37, 5187.

27 P. Zhang, M. Tang, F. Gao, B. Zhu, S. Fu, J. Ouyang, Z. Zhao, H. Wei, J. Li, P. P. Shum and D. Liu, J. Lightwave Technol., 2015, 33, 3332.

28 M. Shatnawi, A. M. Alsmadi, I. Bsoul, B. Salameh, G. A. Alna'washi, F. Al-Dweri and F. El Akkad, J. Alloys Compd., 2016, 655, 244.

29 P. Mahala, A. Kumar, S. Nayak, S. Behura, C. Dhanavantri and O. Jani, Superlattices Microstruct., 2016, 92, 366.

30 Y. Kumar, A. Sahai, S. F. Olive-Méndez, N. Goswami and V. Agarwal, Ceram. Int., 2016, 42, 5184. 
31 P. Fageria, S. Gangopadhyay and S. Pande, $R S C$ Adv., 2014, 4, 24962.

32 S. Fabbiyola, L. John Kennedy, U. Aruldoss, M. Bououdina, A. A. Dakhel and J. JudithVijaya, Powder Technol., 2015, 286, 757.

33 M. Jay Chithra, K. Pushpanathan and M. Loganathan, Mater. Manuf. Processes, 2014, 29, 771.

34 G. Vijayaprasath, R. Murugan, T. Mahalingam and G. Ravi, J. Mater. Sci.: Mater. Electron., 2015, 26, 7205.

35 A. A. Jacob, L. Balakrishnan, K. Shambavi and Z. C. Alex, RSC Adv., 2017, 7, 39657.

36 P. K. Sharma, R. K. Dutta and A. C. Pandey, J. Colloid Interface Sci., 2010, 345, 149-153.

37 T. Bulsgen, M. Hilgendorff, S. Irsen, F. Wilhelm, A. Rogalev, D. Goll and M. Giersig, J. Phys. Chem. C, 2008, 112, 24122417.

38 H. Hao, M. Qin and P. Li, Structural, optical, and magnetic properties of Co-doped $\mathrm{ZnO}$ nanorods fabricated by a facile solution route, J. Alloys Compd., 2012, 515, 143-148.

39 M. Tortosa, M. Mollar, B. Marí and F. Lloret, Optical and magnetic properties of $\mathrm{ZnCoO}$ thin films synthesized by electrodeposition, J. Appl. Phys., 2008, 104, 033901.

40 J. Iqbal, R. A. Janjua and T. Jan, Int. J. Mod. Phys. B, 2014, 28, 1450158.

41 O. D. Jayakumar, J. Cryst. Growth, 2007, 300, 358.

42 J. H. Yang, L. Y. Zhao, X. Ding, L. L. Yang, Y. J. Zhang, Y. X. Wang and H. L. Liu, Mater. Sci. Eng., B, 2009, 162, 143-146.
43 D. Rubi, J. Fontcuberta, A. Calleja, L. Aragones, X. G. Capdevila and M. Segarra, Phys. Rev. B: Condens. Matter Mater. Phys., 2007, 75, 155322.

44 S. Deka, R. Pasricha and P. A. Joy, Phys. Rev. B: Condens. Matter Mater. Phys., 2006, 74, 033201.

45 A. Chanda, S. Gupta, M. Vasundhara, S. R. Joshi, G. R. Mutta and J. Singh, RSC Adv., 2017, 7, 50527.

46 J. Hu, F. Gao, Z. Zhao, S. Sang, P. Li, W. Zhang, X. Zhou and Y. Chen, Appl. Surf. Sci., 2016, 363, 181.

47 R. N. Ali, H. Naz, J. Li, X. Zhu, P. Liu and B. Xiang, J. Alloys Compd., 2018, 744, 90.

48 R. N. Aljawf, F. Rahman and S. Kumar, Mater. Res. Bull., 2016, 83, 108.

49 Y. Xu, R. Zhang, J. Qian, H. Wang, P. Wang and S. Ye, Mater. Des., 2018, 149, 81.

50 H. Li, X. Liu and Z. Zheng, J. Magn. Magn. Mater., 2014, 372, 37.

51 X. Xin, N. Zhong, Q. Liao, Y. Cen, R. Wu and Z. Wang, Biosens. Bioelectron., 2017, 91, 623.

52 S. Y. Yang, Y. F. Chen, H. E. Horng, C.-Y. Hong, W. S. Tse and H. C. Yang, Appl. Phys. Lett., 2002, 81, 4931.

53 H. E. Horng, C.-Y. Hong, S. Y. Yang and H. C. Yang, Appl. Phys. Lett., 2003, 15, 2434.

54 C.-Y. Hong, S. Y. Yang, H. E. Horng and H. C. Yang, J. Appl. Phys., 2003, 94, 3849.

55 N. Zhong, M. Zhao, L. Zhong, Q. Liao, X. Zhu, B. Luo and Y. Li, Biosens. Bioelectron., 2016, 85, 876.

56 K. Bremer, T. Reinsch, G. Leen, B. Roth, S. Lochmann and E. Lewis, Sens. Actuators, A, 2017, 256, 84. 